\title{
On the two-dimensional maps of the distribution of interstellar extinction
}

\author{
A.V. Loktin $\star$ \\ Astronomical observatory of the Ural Federal University, 51 Lenin Street, Yekaterinburg 620083, Russia
}

Received 2013 Apr 3, accepted 2013 Jun 7

Published online 2013 Oct 1

Key words dust, extinction - techniques: photometric

A modified version of the method for determining the mean interstellar extinction and constructing the maps of its distribution based on $J H K s$ photometry is proposed. The extinction for each star of the sample is estimated from the $Q$-index by linearly approximating the line of unreddened stars on the $Q_{J H K}-(J-H)$ diagram. The $J$-band extinction can be calculated by the equation $A_{J}=2.68\left[(J-H)+0.03-1.18 Q_{J H K}\right]$.

\section{Introduction}

Interstellar extinction is a serious problem to face when estimating the parameters of stars and star clusters located in regions of ongoing star formation. Such objects are easier to observe in the infrared, where extinction is weaker than in the optical part of the spectrum. However, estimating extinction from color excesses in the widely used $J H K s$ system is hampered by the fact that reddening lines in two-color diagrams and its analogs run almost parallel to the intrinsiccolor sequence for this photometric system. We hope that extinction maps may help to simplify the task, especially in the cases of high differential reddening. For a comprehensive review of the methods used to construct such maps see Majewski, Zasovski \& Nidever (2011). These methods of extinction map construction can be summarized as follows:

1. Methods based on intrinsic colors of stars:

- the NICE method and its modifications (Lada et al. 1994),

- the RJCE method proposed by Majewski et al. (2011), where Spitzer photometry (GLIMPSE) is used in addition to $J H K s$.

2. Dust emission in the infrared.

3. Star counts (anti-correlation with absorption).

As usual, every method has its own drawbacks. The NICE method uses a single value of intrinsic color $(H-$ $K s)_{0}$ for all stars, which leads to substantial extra dispersion of reddening estimates in each element of the map (pixel). The Ks-band magnitudes on which this method relies are poorly determined compared to the $J$ - and $H$-band magnitudes, at least in the 2MASS PSC (Skrutskie et al. 2006). The RJCE method is based on the measurements by SpitzerIRAC, which are available for a limited part of the sky. The

\footnotetext{
^ Corresponding author: Alexhander.loktin@usu.ru
}

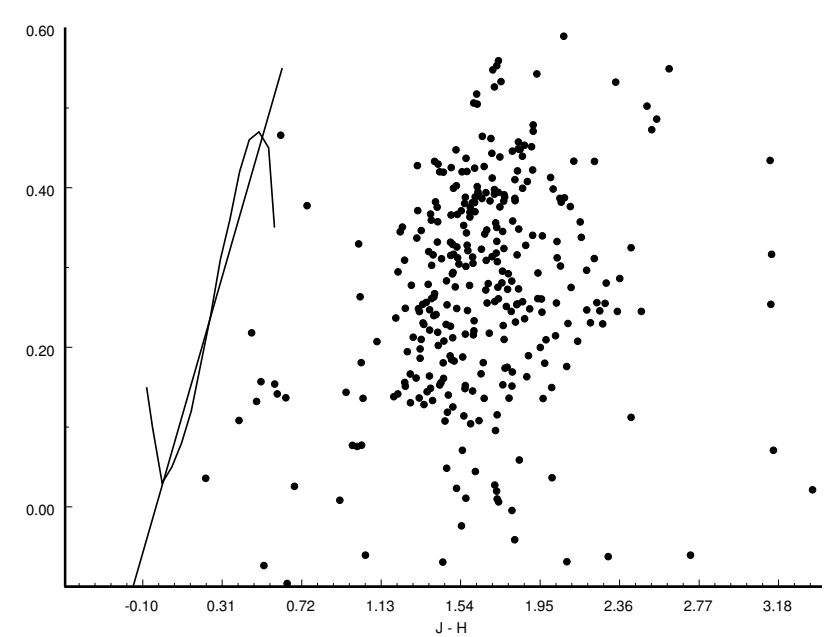

Fig. 1 The $Q_{J H_{K}}-(J-H)$ diagram for a small area surrounding the open cluster NGC 6611 with the unreddened sequence and approximating line superimposed.

results of star counts are difficult to express in terms of extinction values. However, $J H K$ photometry can be used a little more efficiently, and this is the goal of present paper.

\section{The method}

Here we propose a modification of the NICE method, which reduces some of the shortcomings of the original technique, and use our version of the map construction method to compose the extinction map in the area of the NGC 6611 star forming region. Recall that the NICE method assumes that intrinsic color indices $(H-K s)_{0}$ of most of the stars considered lie in a narrow interval and can be replaced by some mean value. However, the most accurate J-band photometry, is of little use in this method. To remedy this shortcoming, we compute intrinsic colors from the $Q_{J H K}$-index values 


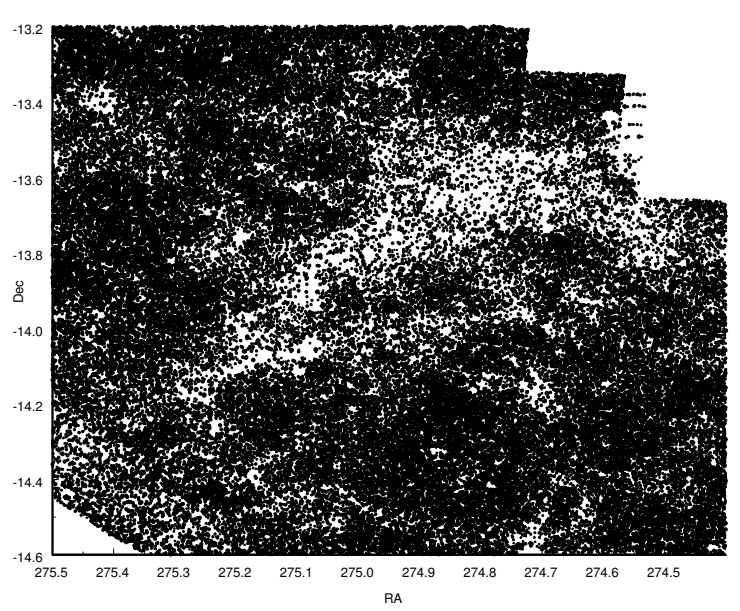

Fig. 2 2MASS based star chart of the area of NGC 6611.

$\left(Q_{J H K}=(J-H)-1.95(H-K s)\right)$ with the color excess ratio adopted from Bessel \& Brett (1988).

Figure 1 shows the $Q-(J-H)$ diagram for an area surrounding the open cluster NGC 6611 together with our unreddened sequence based on the data for several nearby open clusters with good estimates of interstellar reddening. The non-uniqueness of the $Q-(J-H)_{0}$ relation does not allow $(J-H)_{0}$ to be estimated directly from the observed $Q_{J H K}$, and to this end we use the straightline fit of the unreddened sequence, which is also shown in Fig. 1. The straight line deviates from the curve by up to 0.2 for the worst cases of bluest and reddest stars, however, the deviation does not exceed $0 .{ }^{\mathrm{m}} 05$ for A- and F-type stars, which are most numerous among those observed in the sky. The equation of the straight line considered implies the following formula for interstellar extinction: $A_{J}=$ $2.68\left[(J-H)+0.03-1.18 Q_{J H K}\right]$. Hereafter we refer to this extinction calculation method as NICE-Q.

To compare the three methods (NICE, NICE-Q and RJCE), we construct the interstellar extinction map for the NGC 6611 star-forming region. Both 2MASS and GLIMPSE data are available for this area, and we prepared a special 2MASS+GLIMPSE sample within a $1.3 \times 1.3$ field centered in the open cluster NGC6611. The sample is composed only of stars with photometry available in all three 2MASS $(J H K s)$ bands and in the $3.6 \mu \mathrm{m}$ and $4.5 \mu \mathrm{m}$ Spitzer-GLIMPSE bands (Benjamin et al. 2003). For such a sample the dispersion of extinction values in every pixel of the map is determined by the method employed rather than by the characteristics of the sample. Each pixel of the map has a size of $1.2 \times 1 ! 2$, which is more of less equal to the resolution of the map, the size of the map equals to $60 \times 60$ pixels area.

The sample from the 2MASS PSC initially consists of 75468 stars with each pixel of the map typically containing 10 to 40 stars. However, the stars ( $5 \%$ of the sample) with $Q_{J H K}>0.55$ and $<-0.05$ were rejected, because these parameter values may be regarded as abnormal and due to

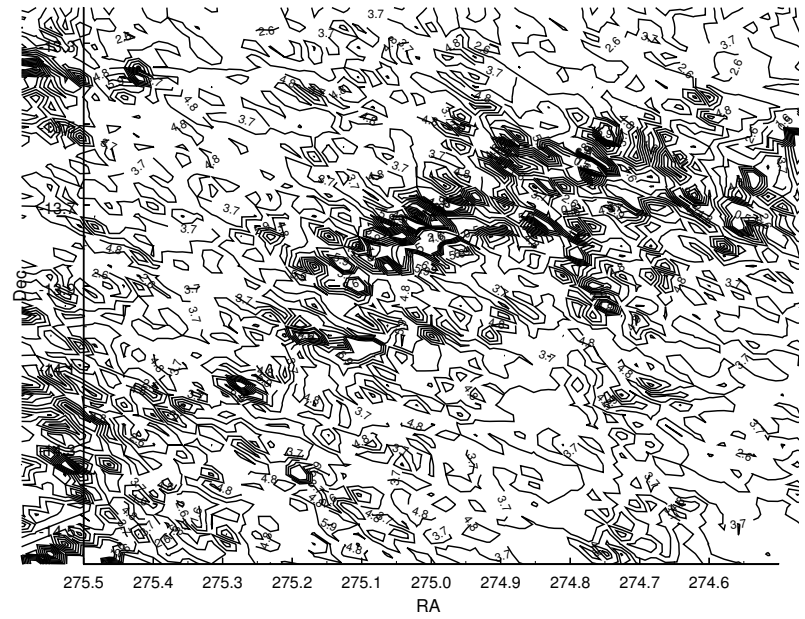

Fig. 3 Distribution of pixel-averaged extinction values in the NGC 6611 region.

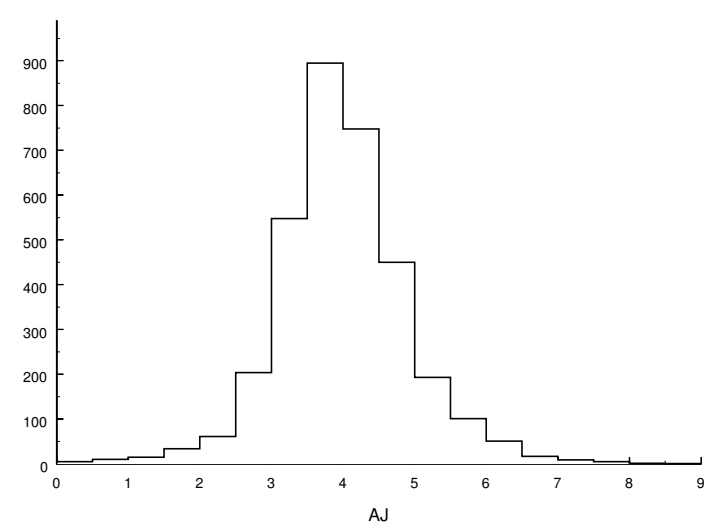

Fig. 4 Distribution of mean extinction values $A_{J}$ determined by the NICE-Q method for the pixels of the area considered.

some stellar peculiarities or large photometric errors (see Fig. 1). Our former experience tells us that this rejection allows not to consider any quality flags in 2MASS. Pixels containing less than two stars are not used in the construction of the map. Such pixels are usually located in the regions of strongest extinction. For each star in a pixel we determine its individual extinction and then compute the weighted mean by averaging extinction values over all stars inside the pixel with the weights proportional to the quality of photometric data.

Figure 2 shows the sky distribution of stars of our sample, and Fig. 3 shows the resulting extinction map obtained using the NICE-Q method. A close correlation between stellar densities and absorption is immediately apparent. The distortion of the shape of the boundary is determined by the available data from the Spitzer-GLIMPSE survey.

Figure 4 shows the distribution of pixel extinction values in the entire area. Extinction is distributed non-uniformly in the area and is very strong, varying around the $A_{J}=4^{\mathrm{m}}$ and reaching as high as $A_{J}=9^{\mathrm{m}}$ in some locations. The latter 

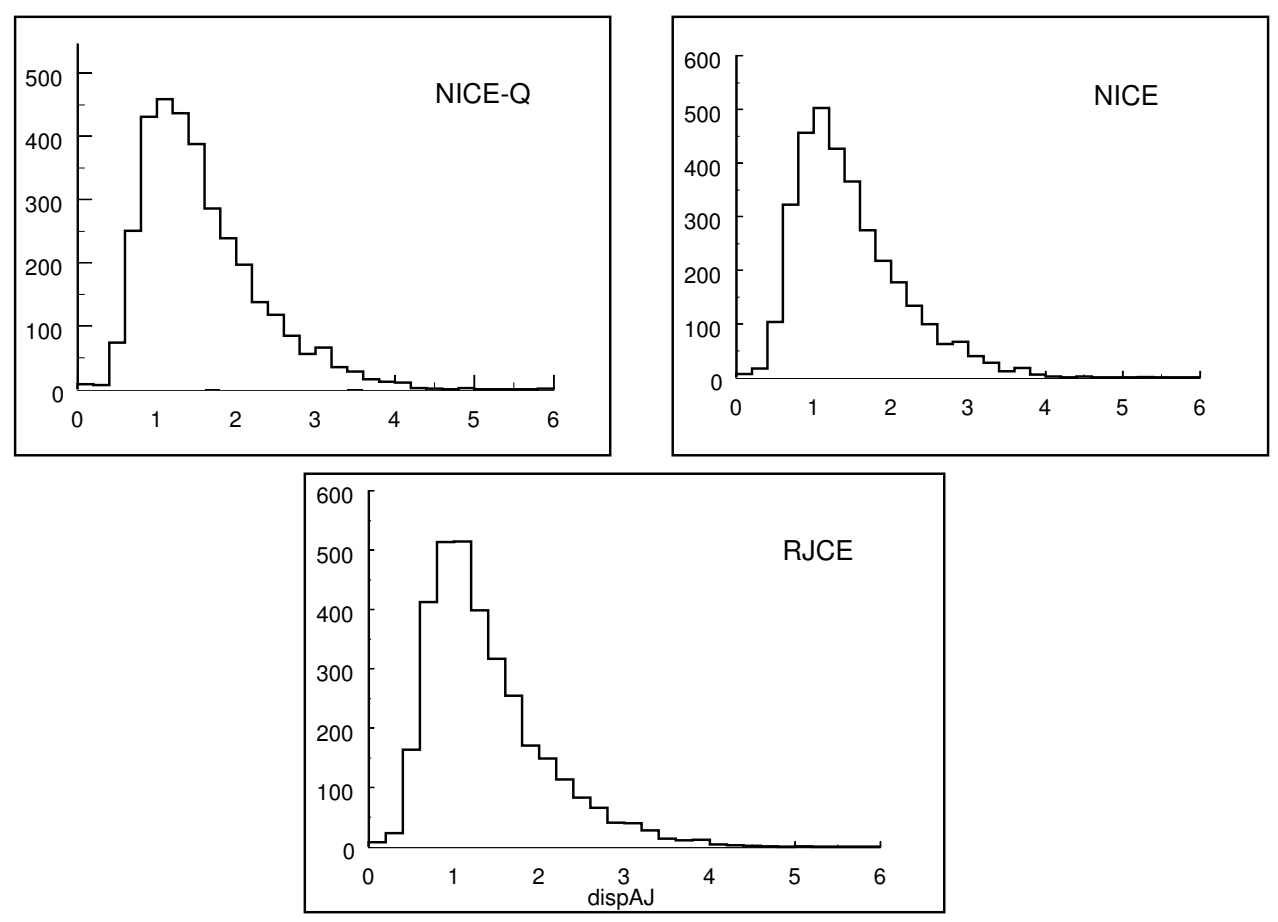

Fig. 5 Comparison of the distributions of dispersions of pixel extinction values determined using three methods.

value characterizes the limiting extinction for the 2MASS data in this direction. A comparison of three extinction maps constructed using three methods (NICE, RJCE and NICE-Q) shows that the mutual correlation coefficients are equal to 1.0 .

The capabilities of the three methods can be compared by computing the dispersions of pixel extinction values. The obvious idea is that for the same sample the smaller the dispersion of extinction estimates for all stars located in the same pixel, the better is the resulting precision of extinction map. Figure 5 shows the distribution of dispersions of extinction estimates for stars in the area considered, determined by three methods separately. All three distributions have nearly the same width, which leads us to conclude that these methods have nearly the same accuracy. The fact that distributions differ little from each other indicates that they reflect the real distribution of extinction values and are not determined by observational errors, which therefore have only a minor effect on the construction of extinction maps.

The RJCE method is now of little practical importance given its dependence on Spitzer-IRAC observations covering only part of the sky.

\section{Discussion}

Our results lead us to conclude that

1. the NICE-Q method has the smallest errors for the red and blue stars and is not based on $K s$-band photometry, although the latter is used to compute $Q$;
2. because of the high density of the 2MASS catalogue it can be used to construct extinction maps for typical star forming regions with a resolution of about one arcmin and penetrate dusty regions up to extinction levels of $A_{V}=25^{\mathrm{m}}$. The deeper UKIDSS photometric data combined with elimination of nearby dwarfs with the help of proper motions will perhaps provide better results both in terms of resolution and accuracy, because of much greater sample sizes.

We finally have three nearly equally efficient methods for constructing extinction maps based on stellar photometry data.

Acknowledgements. This work is supported by the Russian federal task program "Research and Operations on Priority Directions of Development of the Science and Technology Complex of Russia for 2007-2013" (contract 14.518.11.7064).

\section{References}

Benjamin, R. A., Churchwell, E., Babler, B. L., et al. 2003, PASP, 115,953

Bessel, M., \& Brett, J. 1988, PASP, 100, 1134

Lada, C. J., Lada, E. A., Clemens, D. P., \& Bally, J. 1994, ApJ, 429, 694

Majewski, S. R., Zasowski, G., \& Nidever, D. L. 2011, ApJ, 739, 25

Skrutskie, M. F., Cutri, R. M., Stiening, R., et al. 2006, AJ, 131, 1163 